\title{
Challenges in a Technology-based Support Care Intervention among Asian American Breast Cancer Survivors
}

Eun-Ok Im, PhD, MPH, FAAN, Professor, ${ }^{1}$ Wonshik Chee, PhD, Associate Professor ${ }^{1}$ Yun Hu, PhD, Visiting Scholar, ${ }^{1}$ Sangmi Kim, PhD, Post-doctoral Scholar, ${ }_{1}^{1}$ Hanna Choi, PhD, Visiting Scholar, ${ }^{1}$ Yuko Hamajima, MSN, Interventionist, ${ }^{1}$ Eunice Chee, BSE, Research Assistant ${ }^{2}$

${ }^{1}$ School of Nursing, Duke University, North Carolina, USA/ ${ }^{2}$ School of Engineering, North Carolina State University, North Carolina, USA

\section{INTRODUCTION}

Cultural tailoring of technology-based interventions began to be adopted, tested, and widely used in recent years

incorporate specific cultural aspects of racial/ethnic minority groups

serve the special needs of a racial/ethnic minority group more efficiently

Little is still known about practical issues in implementing a culturally tailored

technology-based intervention in health care fields

Only a few publications have discussed the general issues in Internet-based research.

focused on methodological issues studies using the Internet as a data collection method.

\section{OBJECTIVE}

Discuss the challenges that the research team encountered in a culturally tailored technology-based support care intervention among Asian American breast cancer survivors.

\section{THE STUDY: THE BASIS FOR DISCUSSION ON}

Examine the efficacy of a culturally tailored technology-based intervention on improving survivorship of Asian American breast cancer patients

A randomized repeated measures pretest/posttest control group study

intervention group: use the program and the website on breast cancer by the American Cancer Society (ACS) for 3 months

control group: use only the ACS website for 3 months

Participants:

target:330 Asian American breast cancer survivors

three sub-ethnic groups : Chinese, Koreans, and Japanese

Instruments

background factors and disease factors

the Support Care Needs Survey-34 Short Form

the Memorial Symptom Assessment Scale-Short Form

the Functional Assessment of Cancer Therapy Scale-Breast Cancer

\section{METHODS}

\section{Content Analysis:}

the research team members kept the records of research team meetings, and wrote research diaries on challenges in the study;

the meeting minutes and research diaries were analyzed using the content analysis by Weber;

27 Individual words in the minutes and diaries were used as the unit of analysis, and coding was done using a line-by-line coding method; and

categories representing practical issues were made, and six themes were extracted from the codes

\section{RESULTS}

The Challenges found in the research process were extracted into six themes (table1)

\section{FINDINGS \& SUGGESTIONS}

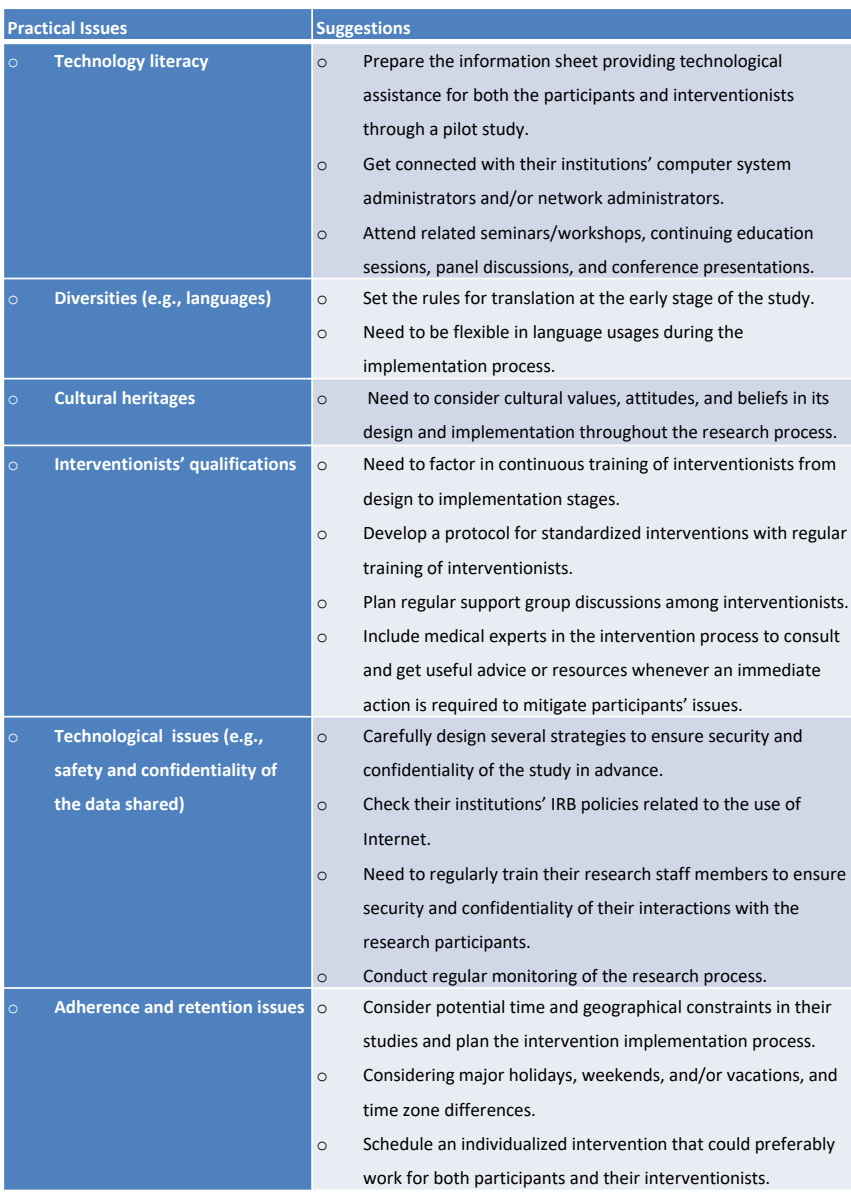

SUGGESTIONS FOR FUTURE RESEARCH

$>$ Researchers need to

$>$ be prepared with detailed step-by-step information sheet for the participants and interventionists in the use of a culturally-tailored technology based interventions;

$>$ set the rules for translation at the early stage of the study and need to be flexible in language usages during the implementation process;

$>$ consider cultural barriers in implementing their technology-based interventions;

$>$ factor in continuous training of interventionists from design to implementation stages to successfully provide participants with appropriate emotional and informational support; \& carefully design several strategies to ensure security and confidentiality of the study in advance; and

consider potential time and geographical constraints in studies

\section{CONCLUSIONS}

The study supports that culturally-tailored technology-based interventions would work very well among racial/ethnic groups, but future researchers need to consider several practical issues in designing and implementing culturally-tailored technology-based interventions. 